\title{
Acute HIV revisited: new opportunities for treatment and prevention
}

\author{
Christopher D. Pilcher, ${ }^{1}$ Joseph J. Eron Jr., ${ }^{1}$ Shannon Galvin, ${ }^{1}$ Cynthia Gay, ${ }^{1}$ and Myron S. Cohen ${ }^{1,2}$ \\ 1Department of Medicine and 2Department of Epidemiology, University of North Carolina at Chapel Hill, Chapel Hill, North Carolina, USA.
}

\begin{abstract}
Inability to recognize incident infection has traditionally limited both scientific and public health approaches to HIV disease. Recently, some laboratories have begun adding HIV nucleic acid amplification testing to HIV diagnostic testing algorithms so that acute (antibody-negative) HIV infections can be routinely detected within the first 1-3 weeks of exposure. In this review article, we will highlight critical opportunities for HIV treatment and prevention that are presented by these diagnostic strategies.
\end{abstract}

\section{Acute HIV infection}

The natural history of HIV infection encompasses an acute/primary phase that lasts months, followed by an early/clinically latent phase that typically lasts 3-10 years, and ultimately by the immune collapse characterized by AIDS. "Acute" HIV infection best describes the interval during which HIV can be detected in blood serum and plasma before the formation of antibodies routinely used to diagnose infection. During this time, high levels of viremia and shedding at mucosal sites can be demonstrated, because HIV replication is unrestrained by immune responses. Approximately 30 days after infection, early virus-specific immune responses are mounted, with subsequent reduction of viremia. After 4-6 months, viral and host factors combine to determine a new pseudo-steady state of viremia (or virologic "set point") for each patient, heralding the beginning of the long clinical latency experienced by patients infected by HIV.

Recognition of acute HIV infection is important for several reasons. First, acute HIV provides a unique view of HIV transmission and pathogenesis, including early host-virus interactions that require further study. Second, prevention strategies directed at subjects with acute HIV infection may have great impact. Third, very early recognition may allow for HIV treatment that could alter the natural history of disease, or even eliminate infection.

\section{Acute HIV revisited: new possibilities for early detection Despite infection of nearly 60 million individuals worldwide with HIV, fewer than 1,000 cases have been diagnosed in the first month of infection, primarily because of a lack of a specific and recogniz- able acute retroviral syndrome. About half of people with acute HIV infection develop headache, fever, myalgias, anorexia, rash, and/or diarrhea (1-3) after an incubation period of around 14 days $(1,4-6)$. Symptoms are generally minor and last days to weeks (1). Genital or oral ulcers may be present, and coinfections with other sexually transmitted pathogens (e.g., herpes simplex virus, gonor- rhea, syphilis, hepatitis viruses) are common (2,7-9). The latter observation suggests common cotransmission of HIV and other sexually transmitted disease (STD) pathogens.}

Nonstandard abbreviations used: antiretroviral therapy (ART); CC chemokine receptor 5 (CCR5); CXC chemokine receptor 4 (CXCR4); sexually transmitted disease (STD); simian human immunodeficiency virus (SHIV); voluntary counseling and testing (VCT).

Conflict of interest: The authors have declared that no conflict of interest exists.

Citation for this article: J. Clin. Invest. 113:937-945 (2004)

doi:10.1172/JCI200421540.
Since acute retroviral syndromes mimic many common febrile illnesses, including infectious mononucleosis, influenza, malaria, and rickettsial diseases $(1,10)$, the true diagnosis (acute HIV) is rarely considered at an initial patient encounter $(1,10-12)$. The diagnostic challenge in acute HIV infection is made more difficult by the fact that routine HIV antibody tests will typically remain negative for 1-2 weeks beyond the onset of acute retroviral symptoms (26-35 days following initial infection) $(5,13)$; additional virus-specific diagnostic tests (e.g., HIV p24 antigen ELISA and HIV nucleic acid amplification assays) are needed to detect HIV infection prior to the appearance of antibodies (Figure 1). While HIV nucleic acid amplification assays are now extremely sensitive and can reliably detect HIV by days 9-11 of infection (13, S1), they are vulnerable to false-positive rates as high as $1 \%$. Such tests remain relatively expensive and have not traditionally been used for routine clinical HIV screening.

The blood-banking industry, however, combines antibody and HIV RNA testing to protect the blood supply. Blood banks submit all donations to routine antibody testing; antibody-positive donations are discarded first. They then combine aliquots from a number of antibody-negative donations, to create a specimen pool. The pools are then screened for HIV RNA. An individual donation is therefore only tested for HIV RNA if its pool first screens positive. In this way, only very few individual specimens are tested for HIV RNA - increasing throuput, decreasing costs, and dramatically reducing false-positive results (Figure 2; refs. 14, S2). Pooling strategies for HIV detection in clinical testing have recently been piloted in Swiss (15), Indian (16), and US (14) clinical-HIV-testing populations. The results suggest that HIV RNA testing - if based on pooling of antibody-negative specimens - can efficiently identify acute HIV infections in clinical testing. Especially in clinicaltesting populations expected to contain low numbers of acute, antibody-negative infections, pooling affords remarkable increases in cost efficiency and predictive value. Clinical studies have clearly indicated that voluntary counseling and testing (VCT) enhanced to incorporate HIV RNA screening can be both clinically (14) and economically feasible $(14,16)$.

Furthermore, studies using various detection strategies (including individual tests for HIV RNA or p24 antigen, as well as pooling strategies) have revealed that acute HIV is not as rare in clinical-testing populations as is commonly assumed. Pincus and colleagues (12) found that five (1.0\%) of 511 consecutive urgent-care-center attendees with "any viral symptoms" in Boston had acute HIV infection confirmed by seroconversion. Rosenberg and colleagues 


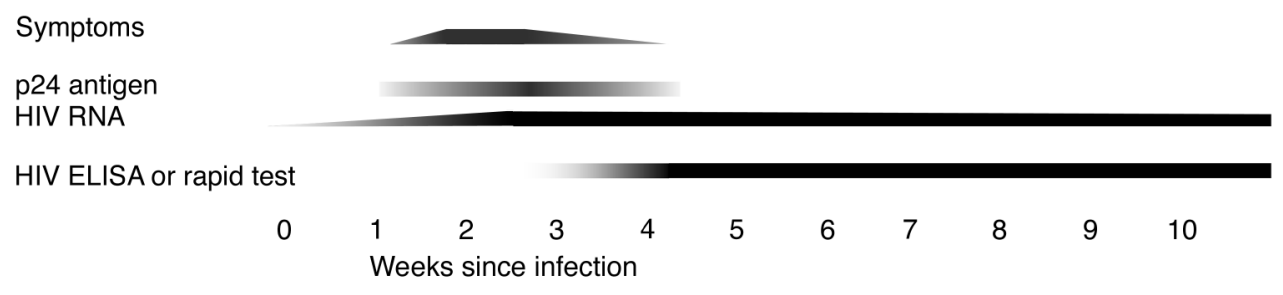

Figure 1

Acute HIV diagnostic timeline. Symptoms, when present, typically occur around 2 weeks after infection. Viremia is detectable prior to symptoms in the form of HIV p24 antigen (detectable by ELISA) or HIV RNA (detectable by even more sensitive nucleic acid amplification). While viremia reaches extremely high levels in the month or two following infection, p24 antigen typically becomes undetectable shortly before seroconversion, because of the formation of early antibody-antigen complexes. A secondary antibody-negative, p24 antigen-negative period is sometimes observed.

examined specimens submitted for heterophile antibody testing for infectious mononucleosis in a university hospital and found that at least four $(0.8 \%)$ of 536 sera examined were from acutely HIV-infected patients (11). Three (0.3\%) of 1,000 emergency-department attendees with fever in North Carolina (17) and six (0.3\%) of 2,300 consecutive, unselected emergency-department attendees in Baltimore (18) had antibody-negative acute HIV. In hyperendemic areas and among patients with STDs, acute HIV infections can be detected even more frequently: 58 (1.2\%) of 4,999 patients at an Indian STD clinic had acute HIV (7), and 23 (2.5\%) of 928 STD clinic attendees in Lilongwe, Malawi, were found to be acutely HIV-infected (2). While the overall percentage of individuals with acute HIV infection in these studies, in both low- and high-prevalence areas, may appear low, a striking finding was that HIV antibody tests have missed more than $3 \%$ of the detectable HIV infections in each testing population. In fact, in Pincus et al.'s and Rosenberg et al.'s studies, antibody-negative infections represented more than a third of all detectable infections among patients presenting for care with apparent viral symptoms. Together, these results indicate that adding HIV RNA screening to current diagnostic screens can commonly identify acutely infected patients and substantially improve the overall sensitivity of HIV testing for infection.

The North Carolina experience. In collaboration with the state of North Carolina, we have developed the Screening and Tracing Active Transmission (STAT) program, which incorporates HIV RNA screening into all publicly funded HIV testing performed for HIV VCT by the central State Laboratory of Public Health (approximately 120,000 specimens are processed per year). HIV RNA screening has directly increased the number of cases identified by $4 \%$ over antibody testing alone, false-positive results have been rare (less than one per 50,000 specimens), and the marginal cost of pooling/HIV RNA testing has remained low (approximately \$2 per specimen) (3). Similar programs are now being launched in other states and abroad. It is therefore critical to revisit the opportunities that detection of acute HIV infection can provide for our understanding of HIV pathogenesis, and for our ability to help patients receive better clinical care and more effective prevention.

\section{The biology of HIV expansion}

Initial expansion of viral reservoirs. HIV's attachment to and entry into cells results from a complex interaction of the HIV envelope proteins gp120 and gp41 with cell surface receptors (CD4 and CC chemokine receptor 5 [CCR5] or CXC chemokine receptor 4 [CXCR4]) that are present on $\mathrm{T}$ cells and/or macrophages (19,
S3-S7). In acute HIV infection resulting from sexual exposure to HIV-1 (Figure 3), the virus successfully traverses the epithelial layer by one of several proposed mechanisms: transcytosis, epithelial cell capture, or exposure to subepithelial target cells through disruptions in mucosal integrity (reviewed in ref. 20). Mucosal DCs express C-type lectins (e.g., DC-SIGN), which can both specifically bind HIV and facilitate its internalization. HIV-1 that is bound and internalized by a DC may allow infection of nearby $\mathrm{CD}^{+} \mathrm{CCR}^{+}$cells and/or efficient presentation of HIV-1 to susceptible target cells after DCs migrate to draining lymph nodes (21, S8). However, simian human immunodeficiency virus-infected (SHIV-infected) memory $\mathrm{CD}^{+}{ }^{+} \mathrm{CCR} 5^{+}$cells have been detected in mucosal tissues of macaques as early as 6 hours after exposure, which suggests the possibility of direct infection (22, S9, S10). Ultimately, HIV-infected CD4 ${ }^{+}$cells can be found in lymphoid tissue within 2 days of infection, and the magnitude of infection present in these tissues is sufficient to account for the subsequent rise in plasma viremia $(23, \mathrm{~S} 11)$.

Initial viremia expands exponentially, with a doubling time of approximately 0.3 days during the first $2-3$ weeks of infection (13, S12-S14). Experimental primate models of mucosal HIV infection have demonstrated that maximal levels of viral expression occur in different tissue compartments sequentially over short periods of time $(22,24,25)$. Viral loads in blood, genital secretions $(26,27)$, and other compartments peak at very high levels around 4 weeks after infection (27) and then decrease in association with the appearance of virus-specific CTL responses $(4,28)$.

The overall composition of the $\mathrm{CD}^{+}$cell population changes during the course of acute HIV infection. Provided the individual does not have another systemic infection, the CD4 cell population consists predominantly of resting (HLA-DR-CD38-) cells (S15-S19). Increasing HIV-1 replication during acute infection leads to a general activation of $\mathrm{CD}^{+}$and $\mathrm{CD}^{+}$cells, including $\mathrm{HIV}-1-$ specific $\mathrm{CD}^{+}$cells. Activated $\mathrm{CD}^{+}$cells are likely to be successfully infected with HIV-1 (29); it is therefore likely that the very cells necessary to orchestrate a robust immune response are targeted for destruction during acute infection.

Host-virus interactions: selection, compartmentalization, and diversification. The host-virus interaction shapes the population of HIV variants in systemic and tissue compartments. The number of replication events that occur during acute HIV infection is extremely large, and the opportunity for host factors to exert pressure on existing or newly mutated viral variants is substantial. Host factors exerting early selective pressure may include the cells initially infected, or microenvironments in the mucosa, submucosa, and lymph nodes, which present a variety of innate host defenses including IFNs and/or other molecules (30, 31, S20). Host selective pressures may either decrease or increase the diversity of the infecting-virus population. For example, most acute infections (97\%) result from HIV variants that use CCR5, regardless of route of infection (32-35, S21-S25), which suggests some form of selection against variants that use CXCR4. 
A

Pooling of aliquots from 90 HIV-seronegative specimens

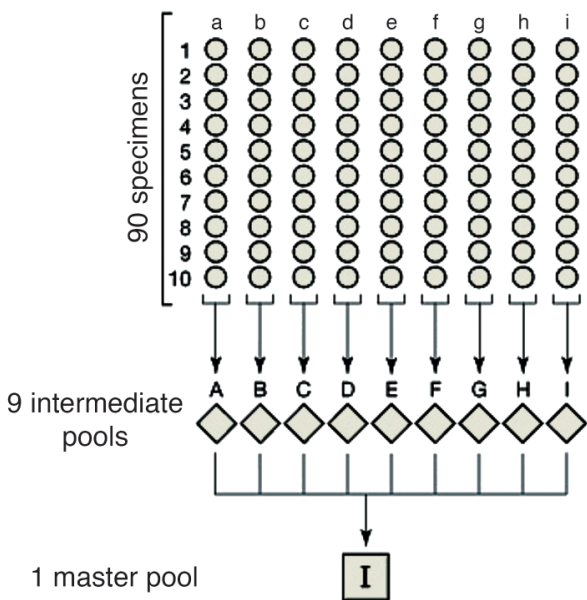

B Resolution testing of master pools

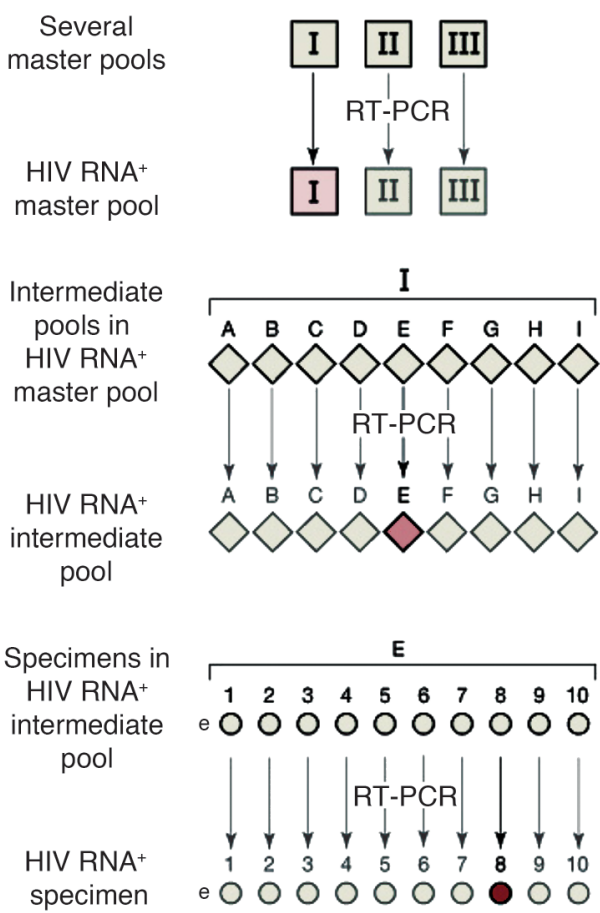

Establishment of infected cell pools in acute HIV infection. While exponential replication of HIV-1 with attendant CD 4 cell activation and loss is the primary dynamic during very early infection, more subtle processes begin at the same time. Small populations of $\mathrm{CD}^{+}$ cells with a resting memory cell phenotype begin to integrate HIV-1 DNA in the chromosome. Such cells are detected relatively early in HIV infection (36), and at least some cells appear to have a long halflife (S26-S28). Two reservoirs of latently infected cells are likely to exist from early in acute infection, the more abundant one having "pre-integration" HIV-1 and the other having integrated, replication-competent HIV. After initiation of antiretroviral therapy (ART), the two cell subpopulations can be distinguished. Cells with preintegration HIV and a shorter half-life decay, revealing a smaller,

\section{Figure 2}

Specimen pooling for HIV RNA testing. The algorithm shown here is that used by the North Carolina State Laboratory of Public Health to increase the predictive accuracy and cost efficiency of the screening of all sera, obtained through HIV voluntary counseling and testing, that are initially antibody-negative for HIV RNA. (A) Illustration of the creation of intermediate and master pools in a 1:10:90 pyramid-style pooling scheme. Only HIV-seronegative specimens are pooled. In each step, $200-\mu \mathrm{l}$ aliquots are drawn off for pooling. The master pool ultimately contains sera from 90 antibody-negative individuals. (B) Illustration of the manner in which pools are screened using HIV RNA amplification testing. Positive results on a master pool trigger testing of intermediate pools and, in the final round of testing, individual specimens. When only a small number of specimens in a population are truly HIV RNA-positive, this procedure results in a dramatic reduction in the number of RNA tests used and virtually eliminates false-positive RNA test results in the final round of testing. Figure reprinted with permission from Journal of the American Medical Association (16).

long-lived reservoir (37). These long-lived cells are invisible to the immune system and have a decay half-life on the order of 48 months (S26). Such slow decay is explainable either by persistence of infected cells or by continued, low-level viral replication (38, S29). Persistence of quiescent but infected cellular reservoirs makes HIV incurable with current interventions.

The precise dynamics of the establishment of this long-lived reservoir during acute HIV infection are unknown. Two clinical trials have quantified reservoirs of HIV in latently infected $\mathrm{CD} 4^{+}$cells in seronegative acutely HIV-infected patients treated with a standard duration of $\operatorname{ART}(39,40)$. Each found that patients started on therapy while they were still HIV antibody-negative had lower levels of latently HIV-infected $\mathrm{CD}^{+}$cells, consistent with the hypothesis that these reservoirs are fully established only toward the end of the acute-infection interval.

The adaptive immune response. Infections with HIV-1 and with related viruses that infect nonhuman primates present a particularly complex interaction with the host immune system, as the primary target cells are critical $\mathrm{CD} 4^{+} \mathrm{T}$ cells, which are themselves essential to a robust pathogen-specific immune response (reviewed in ref. 41).

The initial HIV-1-specific immune response is characterized by the appearance of non-neutralizing antibodies detected by ELISA $2-4$ weeks after infection $(13,42)$. These antibodies serve as the basis for the diagnosis of HIV. However, they have no effect on viral load and appear to exert little selective pressure (43). Neutralizing antibodies are detected 8 weeks or more after infection (43-45), well past the viral-load peak (S30-S33). Neutralizing antibodies seem to place strong immune pressure on the virus, as evidenced by escape variants that appear $(44,45)$ together with an explosion of viral diversity (43). While neutralizing antibodies exert strong selective pressure, they do not appear to have a sustained effect on HIV (or SHIV) RNA levels in plasma, which demonstrates the ease with which HIV-1 escapes from this immune response (45).

Retrovirus-specific CD8 ${ }^{+}$CTLs detectable early after infection are strongly associated with control of viremia in acute infection and established infection (4, 41, 46, 47, S34-S36). CTL escape variants can be identified as early as 4 weeks after acute SHIV infection in macaques (S37), and escape variants can establish HIV infection in vertical transmission $(48, S 38)$. The initial CTL response(s) may be directed against a few epitopes and subsequently broaden during prolonged antigen stimulation (S39). Epitopes targeted during 
$\mathbf{A}$

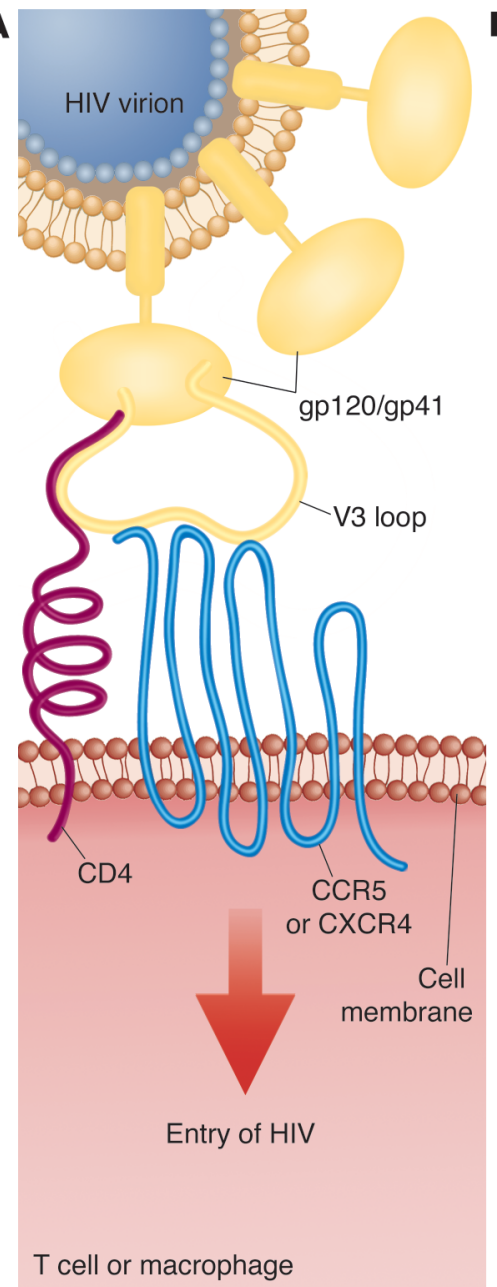

Figure 3

B

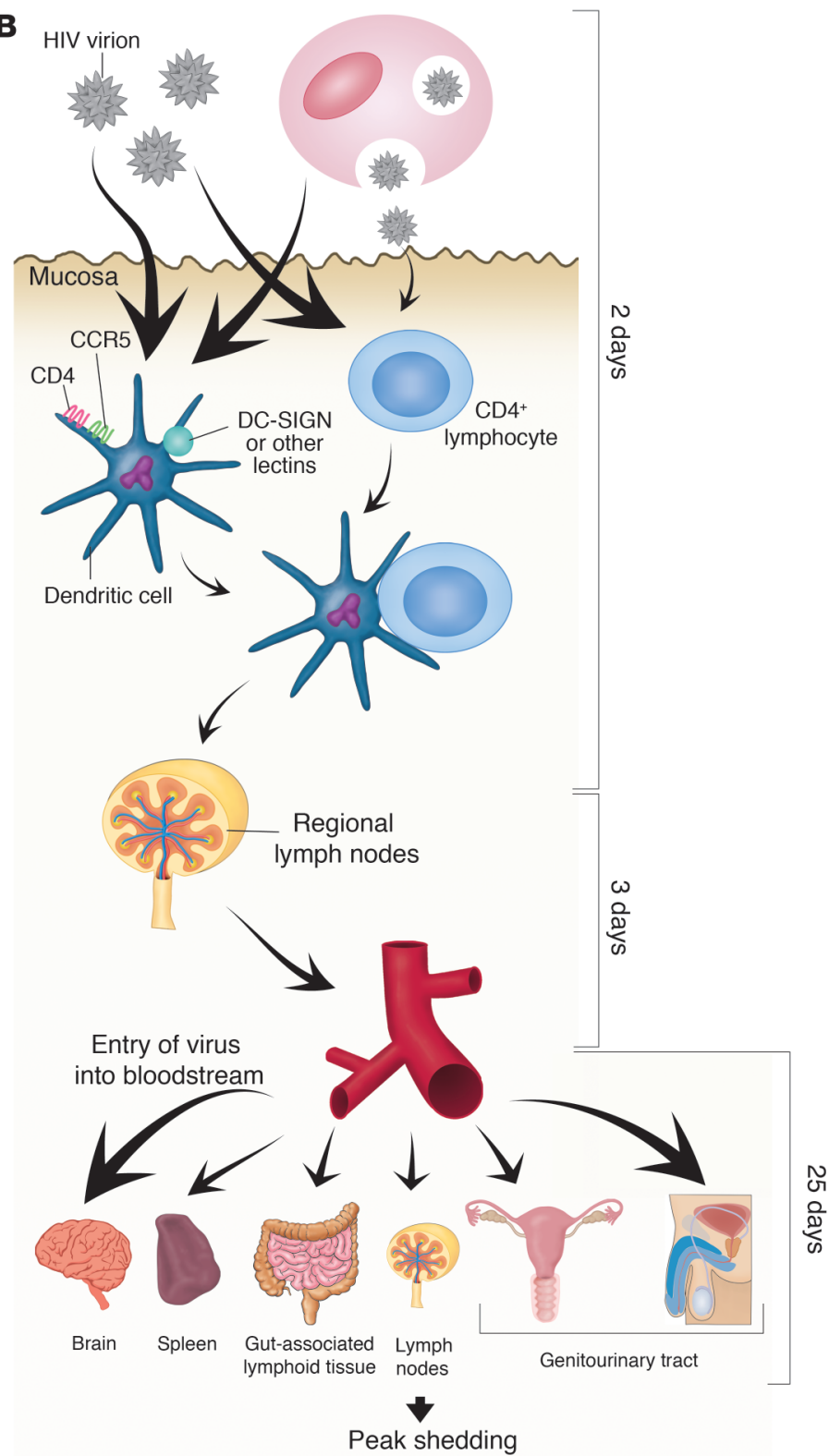

HIV transmission and the establishment of HIV reservoirs. (A) Interactions of HIV envelope glycoproteins, CD4, and CCR5 or CXCR4 coreceptors trigger fusion and entry of HIV. (B) Outline of the sequence and time course of events involved in viral dissemination. Figure adapted with permission from New England Journal of Medicine $(98,99)$.

acute infection often differ from those recognized during chronic infection (S39, S40). The importance of the CTL response in modulating HIV-1 viremia can be inferred by the association of specific HLA types with low HIV RNA levels and slowed disease progression (49-51; S41, S42); by the demonstration that specific HLA-restricted CTLs are associated with better control of SHIV and HIV replication (S43, S44), even in acute infection (S45); and by selection for viral escape $(4, \mathrm{~S} 37)$. However, all initial CTLs are not created equal. Some specific CTLs in the SHIV model select rapidly for escape variants, while others appear to offer little selective pressure (S46-S48). The avidity of the initial CTL responses may be extremely important, and the ease with which virus can escape highly avid responses may dictate the level of control. How- ever, multiple previously unrecognized CTLs may be present (S39), and the sequence of events leading to effective CTL responses during acute HIV infection is not clear. In particular, the potential effects of ART on developing cellular responses is a matter of controversy: in one study, patients in whom ART was initiated before seroconversion demonstrated narrower and lesser-magnitude CTL responses to HIV than did untreated patients (S49).

However, HIV-specific $\mathrm{CD}^{+}{ }^{+}$responses may also be an essential component of the initial adaptive immune response (52, 53, S50) and, in acute infection, may be associated with robust CD8 ${ }^{+} \mathrm{CTL}$ responses $(54, \mathrm{~S} 49)$. In the study cited above, treatment during acute infection either before or after seroconversion led to higher $\mathrm{CD}^{+}$Th cell responses than treatment of chronically infected 


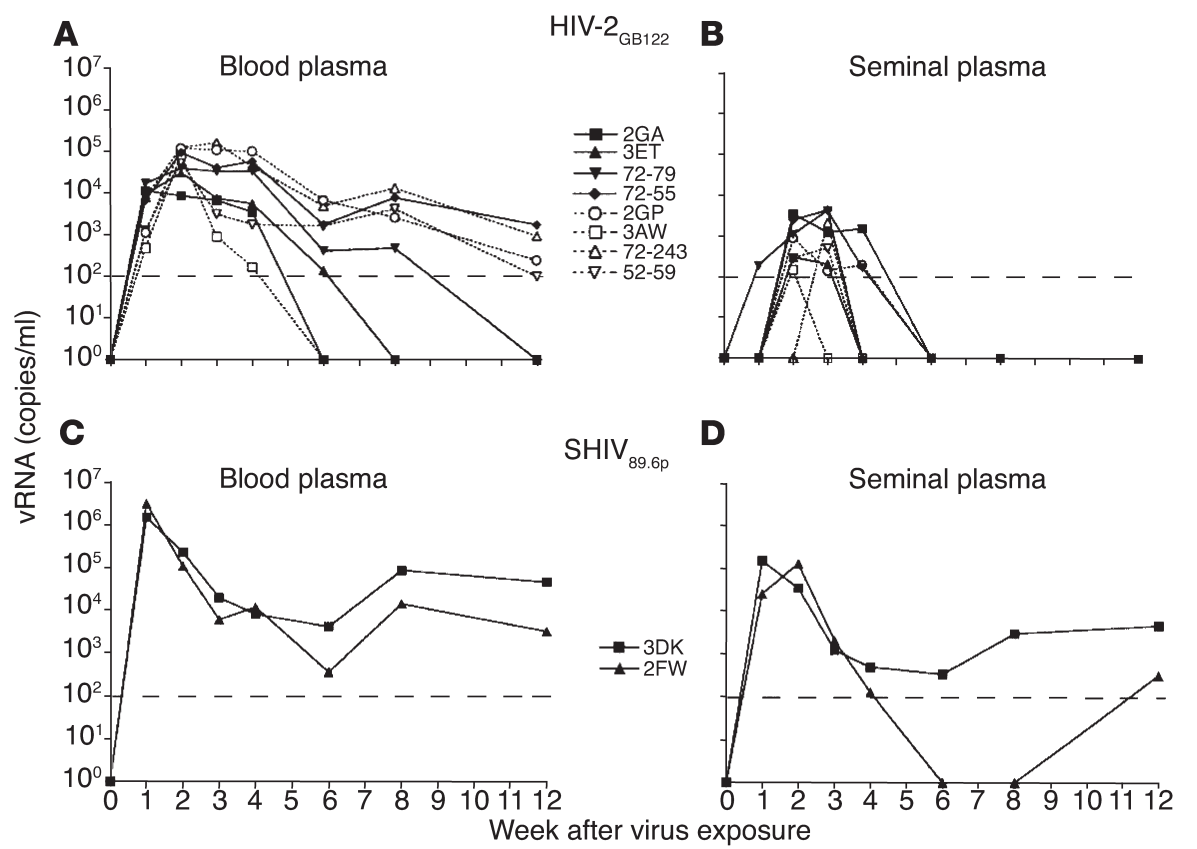

\section{Figure 4}

Similarity in the dynamics of viremia and genital shedding in experimentally HIV- or SHIV-infected macaques. (A and B) Virionassociated RNA (vRNA) levels in blood plasma (A) and seminal plasma (B), observed in pig-tailed macaques during the first 12 weeks after intravenous $(n=4$; filled symbols) or intrarectal exposure $(n=4$; open symbols and dashed lines) to HIV type $2\left(\mathrm{HIV}_{\mathrm{GB} 122}\right)$. (C and D) vRNA levels in blood plasma (C) and seminal plasma (D) through 12 weeks after intravenous exposure $(n=2)$ to simian/HIV $\left(\right.$ SHIV $\left._{89.6 \mathrm{p}}\right)$ are also shown. Peak viremia and peak genital shedding occur simultaneously and resolve over a similar time frame in each model. Figure modified with permission from Journal of Infectious Diseases (83). patients (S49). There is continued debate over the relative importance of quantitative HIV-1-specific CD4 ${ }^{+}$cell responses (S51).

\section{Acute HIV and clinical implications}

A unique role for acute treatment: augmenting host immune control of HIV. Highly potent ART in current use results in significant reversal of disease progression in HIV-infected individuals with advanced disease. Prolonged treatment, however, continues to be limited by drug toxicity, problems with adherence, drug resistance, and cost. To date, the principal rationales for antiretroviral treatment early in acute HIV infection rest on the ability of potent ART (a) to treat some symptomatic patients; (b) to halt viral evolution at a time of minimal viral diversity, prior to viral adaptations to specific host immune responses; (c) to protect developing immune responses from the deleterious effects of sustained HIV viremia; (d) to reduce the viral set point; and (e) to limit the latent pool. Rosenberg and colleagues noted that initiation of ART during the early stages of acute HIV infection led to the development of unusually broad and strong HIV-1-specific Th cell proliferation in response to HIV antigens $(52,54)$. These data support the hypothesis that early targeting of HIV-1-specific T cells by HIV may be an important pathogenic mechanism allowing persistent, high-level viral replication in the host after resolution of acute infection (29) - and that early intervention with ART could boost initial host responses to infection. If true, this would suggest that treatment of acute HIV might serve as a method to augment host immunity and might thus obviate or delay the need for lifelong continuous ART.

Early observations supported a possible role of ART in the management of acute HIV infection. Anecdotes described patients that experienced prolonged periods of controlled viremia in conjunction with strong, HIV-specific cellular immune responses after discontinuing ART initiated during acute infection (55, S52). One of the first prospective studies on structured treatment interruption (STI) in acute HIV infection incorporated the concept of viral rebound as antigenic stimulus to enhance $T$ cell responses. The protocol involved treatment interruption after a minimum of 8 months of undetectable viral load, with therapy reinitiated if the viral load increased to greater than 5,000 RNA copies per milliliter plasma for 3 consecutive weeks, or greater than 50,000 copies at one time (54). Three patients achieved long-term viral suppression after a single treatment cessation. The remainder required re-treatment and a second interruption, after which all individuals experienced transiently suppressed plasma viremia, and two achieved sustained viral suppression. In all cases, interruptions were followed by enhancement in magnitude and breadth of cellular HIVspecific immune responses. This trial lacked a concurrent control population, and a clinically important effect of therapy on HIV RNA levels in patients can only be inferred by comparison with HIV RNA levels previously observed in populations of patients not receiving this therapy, or by observation of delayed rebound of HIV RNA in plasma after a period of relative suppression.

The principal adverse events in early studies have been isolated cases of a retroviral syndrome similar to primary HIV infection, associated with initial discontinuation of therapy (56, S53). Importantly, prolonged observation of the patients studied in the trial described above has shown that initial immune control of HIV replication can wane 6-30 months after removal from therapy, in association with viral rebound to more typical levels of viremia and concurrent evolution of escape mutations to HIV-specific CTL epitopes or neutralizing antibody $(57,58)$. These results emphasize that the benefits of acute treatment may not translate to permanent remission of HIV disease. Still, acute HIV remains the only setting in which the natural history of disease may be altered by the temporary use of ART.

The proving ground for curing HIV. The decay of HIV-infected cell populations in patients receiving potent ART resulted in initial enthusiasm for the "eradication hypothesis" (59). Since then, attempts to cure established HIV infection with antiviral agents have met with absolutely no success. As described above, a subpopulation of HIV-infected $\mathrm{CD}^{+}$lymphocytes avoids viral or immune cytolysis and enters the resting state (S27, S54). This reservoir, invisible to the immune system, appears to have a very low 
decay rate even in the setting of prolonged antiretroviral suppression (S26), though treatment during early infection may accelerate this decay (S28). Addition of cytoreductive chemotherapy (cyclophosphamide) to ART failed to reduce proviral DNA load in lymphatic tissue (S55). Several groups have studied use of global T cell activation agents to perturb latent reservoirs in aviremic, treated patients; IL-2, low-dose OKT3, and/or IFN- $\alpha$-based regimens have shown evidence of decreasing latent reservoirs but have failed to eliminate latent infection $(60,61, \mathrm{~S} 56)$. It is possible, however, that global $\mathrm{T}$ cell activation may induce viral replication and increase the number of susceptible uninfected target cells beyond a threshold that can be contained by ART. Newly described host mechanisms that regulate HIV gene expression involving histone deacetylase 1 (62, S57-S59) may present therapeutic targets for selective derepression of HIV gene expression, to allow outgrowth of quiescent HIV without the pitfalls of global T cell activation.

Regardless of the specific approach, acute HIV infection could be a critical proving ground for eradication strategies, for several reasons. First, it is possible that initiation of intensive ART in very early (antibody-negative) acute HIV can significantly limit the size of the initial latent pool $(39,40)$ - effectively lowering the bar for all strategies that target this reservoir. Second, patients treated in the first 3 weeks of infection may be able to marshal maximal HIV-specific immune responses to facilitate reservoir clearance $(54,63,64)$. Third, in macaques, HIV infection can be prevented when ART is provided for 28 days within 72 hours after exposure (64); this suggests that nascent latent reservoirs can be cleared. Fourth, early acute HIV infection is the only setting in which clinical benefits have been associated with brief ( $<1$ year) and interrupted courses of intensive ART (54).

Individual infectiousness, disease stage, and probability of sexual transmission. The main routes of HIV transmission are sexual, maternal-child, and parenteral. The majority of HIV transmission worldwide occurs through sexual contact (S60) with mucosal exposure to infected genital secretions. Regardless of the route of exposure, in every case transmission of HIV is determined by the infectiousness of the transmitting host and the susceptibility of the exposed person (66).

Determinants of host susceptibility may include CCR5/CXCR4 coreceptor polymorphisms $(67$, S61) and HLA types. Alterations in genital mucosa may also have a profound effect on HIV susceptibility. The presence of STDs is an important risk factor for acquiring $\operatorname{HIV}(66,68,69$, S62). Both genital ulcerative diseases like herpes, syphilis, and chancroid and inflammatory diseases such as gonorrhea increase HIV acquisition $(66,68,69$, S62). Bacterial vaginosis in women has also been shown to be a risk factor for HIV acquisition (70); other factors such as vaginal thinning from estrogen deficiency (S63) and cervical ectopy (S64) affect HIV susceptibility as well. In men, changes in the penile epithelium that occur with circumcision can be protective (71).

Biological factors strongly influence infectiousness. High plasma viral loads correlate with risk of transmission by vertical (S65, S66) and sexual (72) routes. Conditions such as tuberculosis (S67) and malaria (73) that elevate plasma viral loads would therefore be expected to be associated with increased infectiousness. Genital viral load in semen (74-76, S68) or female genital fluids (77) can be increased to a level greater than that of blood plasma viral load by the inflammation caused by STDs $(68,69,75,78,79$, S62). In addition, nutritional deficiencies and hormonal influences in women (S69, S70) can increase shedding of HIV in the genital tract. Such transient elevations of genital or rectal shedding of HIV are considered periods of "hyperinfectiousness."
Disease stage is powerfully associated with individual infectiousness. Higher viral loads correlate with higher transmission probability in patients with advanced disease (80, S71). However, the most striking elevations in HIV viremia (S72, S73) and genitalfluid shedding $(27,81,82)$ occur early in acute infection. Based on a probabilistic model of the relationship between semen viral burden and probability of HIV transmission (83), increases in semen HIV load during acute HIV infection appear sufficient to account for an eight- to 20 -fold increase in the odds of transmission per coital act (27). Other biological influences hypothesized to contribute to elevated individual infectiousness in acute infection include the relative homogeneity in Env (84), the near universal R5 coreceptor usage (35), the absence of antibody in genital fluids (S74), and the frequent presence of other inflammatory or ulcerative STDs that might increase both shedding and partner susceptibility $(7,9,27)$. The notion of cotransmission of STD pathogens suggests one location where acute HIV infection might be best detected: STD clinics. Recent studies have borne out that STD clinic attendees in the US (14), Malawi (2), and India (16) can have a surprisingly high prevalence of acute HIV infection, and Cameron et al. generated compelling evidence for cotransmission of herpes simplex virus and HIV in the late 1980s (85).

Role of acute HIV in epidemic spread. A high proximate risk of secondary HIV transmission has been clearly associated with stage of disease. Secondary infections (from an acutely infected index case to a susceptible partner) have been documented beginning as early as 7-14 days after initial infection, prior to the onset of acute retroviral symptoms in the acutely infected index case (S74). In one study examining sexual risk behavior during and after HIV seroconversion, Colfax and colleagues (86) documented alarming rates of partner change and sexual concurrency among acutely HIVinfected men who have sex with men in California: 39 (59\%) of 66 acutely infected men in the study reported unprotected insertive anal intercourse with additional (HIV-susceptible) partners during the time of acute infection, with no apparent decrease in risk behavior associated with the immediate acute-infection period even in symptomatic patients.

Mathematical models $(87,88)$ have previously suggested that rapid global spread of HIV can be ascribed to hyperinfectiousness in the acute phase of HIV disease; empiric epidemiologic data now strongly support this hypothesis. Retrospective cohort studies of patients infected by sexual contact (89) or blood transfusion (S75) have confirmed significantly increased risk of infection among those exposed to index cases with acute or recent infection. Recently, Wawer and colleagues (80) examined the link between acute disease stage and transmission in a remarkable study of couples in Rakai, Uganda, who were screened for HIV infection and followed as part of an STD treatment trial. An estimated $48 \%$ of all HIV transmission events observed among all serodiscordant couples in the study occurred in situations where the first infected partner had acquired HIV during the preceding 5 months.

The potential magnitude of the contribution of acute hyperinfectiousness to transmission is revealed by two studies examining the phylogenetic relatedness of viral strains in newly infected patients in defined communities. Among 191 newly infected patients in the Swiss HIV Cohort, 29\% shared viral sequences, time of infection, and geography with at least one other acutely infected patient (90). In a similar study in New York, New York, 51 (21\%) of 241 patients were found to be clustered, and acute-to-acute transmission was confirmed by history in nine cases (S76). There are two 
principal alternative explanations for such extensive clustering. The first is efficient, rapid dissemination by acutely infected individuals, leading to serial infections in sexual networks. However, "core transmission" by a small unidentified subpopulation of HIV-positive men or women in the same population could also lead to the same outcome $(91, \mathrm{~S} 77)$. In either scenario, the ability to identify acutely HIV-infected men and women could open a unique window on active HIV transmission taking place in a community, and provide opportunities to interfere with the chain of HIV transmission.

Intervention: targeting prevention to networks with active disease transmission. Traditional surveillance systems, which are based on detection of undifferentiated, prevalent cases of HIV disease, can describe where an epidemic has been, but not where it is going. Because traditional surveillance cannot isolate the context or conditions of HIV incidence, the results cannot describe local forces (socioeconomic, cultural, and geographic) that drive HIV spread in communities (92). Incident STDs can be heavily concentrated in definable social networks and particular locales that are not immediately obvious (93, 94, S78, S79). Identifying local and regional patterns of HIV transmission can allow targeting of high-transmission areas for public health interventions (S60). Reliable information about the locations and correlates of incidence can furthermore ratify information gathered from other sources (e.g., behavioral surveys or cross-sectional serosurveys) and provide early indications of the effectiveness of interventions. The drive to identify regional variation in incidence rates has previously led to development of "detuned" HIV antibody assays that remain negative in some patients with recent infection and low-avidity antibodies; one such assay under evaluation by the Centers for Disease Control and Prevention has proven useful for estimating seroincidence in cross-sectional, population-based samples (95). Unfortunately, the current generation of detuned assays are unable to accurately classify individual patients as having truly recent infection.

One particularly promising approach to HIV control combines identification of acute disease with "network notification," linking case reporting to the tracing of contacts and offering partner counseling and referral services (96). Applying this strategy prospectively to incident HIV could harness classical infection-control techniques that have been successfully used for decades in tuberculosis- and syphilis-eradication campaigns. Modern epidemiologic techniques can supplement the information provided by STD contact tracing. For instance, spatial analysis based on geographic information systems can be applied to incidence data to define high-transmission areas for HIV (S80, S81), and modern social-net- work analysis $(97$, S82) can give a clear understanding of transmission patterns in communities. The effectiveness of any such creative, proactive public health strategies for HIV prevention hinges critically on better understanding the effectiveness of specific interventions - such as partner counseling or ART (given as treatment, postexposure prophylaxis, or pre-exposure prophylaxis) - at reducing disease transmission.

\section{Conclusions}

While compelling evidence suggests the potential importance of detecting acute HIV infection, remarkably little attention has been devoted to this goal. This oversight results from a widespread belief that neither clinical nor serosurveillance methods have much chance of identifying patients with acute HIV infection. Not surprisingly, cogent treatment and prevention strategies for this group have not been developed. However, new methods in populationbased serosurveillance and increased focus on some kinds of STD patients could allow identification of a large number of patients with acute HIV infection. The detection of acute disease will permit exciting research, clinical, and public health opportunities.

\section{Acknowledgments}

This work was supported by the NIH (RO1 MH68686, K23 AI01781, P30 AI50410, RR 00046, RO1 DK49381, [HPTN, std and pathogenesis training grant numbers]). The authors wish to thank Susan Fiscus, Ronald Swanstrom, William Miller, Charles Hicks, and Peter Leone for their scientific contributions targeting acute HIV. We acknowledge the invaluable collaboration of Todd McPherson, Leslie Wolf, and Lou Turner of the North Carolina State Laboratory of Public Health, as well as Evelyn Foust, Del Williams, Judy Owen O'Dowd, Rhonda Ashby, and Todd Vanhoy of North Carolina Department of Health and Human Services' HIV/STD Prevention and Care Branch. The UNC Retrovirology Core laboratory staff deserve special thanks for their dedication to innovation.

Due to space constraints, a number of important references could not be included in this article. Interested readers can find a supplementary reading list at http://www.jci.org/cgi/content/full/ 113/7/937/DC1.

Address correspondence to: Christopher D. Pilcher, CB\#7215, 211A West Cameron Street, University of North Carolina at Chapel Hill, Chapel Hill, North Carolina 27599-7215, USA. Phone: (919) 843-2721; Fax: (919) 966-8928; E-mail: cpilcher@med.unc.edu.
1. Schacker, T., Collier, A.C., Hughes, J., Shea, T., and Corey, L. 1996. Clinical and epidemiologic features of primary HIV infection. Ann. Intern. Med. 125:257-264.

2. Pilcher, C.D., et al. 2004. Frequent detection of acute primary HIV infection in men in Malawi. AIDS. 18:1-8.

3. Pilcher, C.D., et al. 2004. The "Screening and Tracing Active Transmission” (STAT) program: realtime detection and monitoring of HIV incidence. Program and abstracts: 11th Conference on Retroviruses and Opportunistic Infections. San Francisco, California, USA. Abstr. 20

4. Borrow, P., et al. 1997. Antiviral pressure exerted by HIV-1-specific cytotoxic T lymphocytes (CTLs) during primary infection demonstrated by rapid selection of CTL escape virus. Nat. Med. 2:205-211.

5. Lindback, S., et al. 2000. Diagnosis of primary HIV1 infection and duration of follow-up after HIV exposure. Karolinska Institute Primary HIV Infection Study Group. AIDS. 14:2333-2339.

6. Pilcher, C.D., et al. 2001. Sexual transmission during the incubation period of primary HIV-1 infection. JAMA. 286:1713-1714.

7. Bollinger, R.C., et al. 1997. Risk factors and clinical presentation of acute primary HIV infection in India. JAMA. 278:2085-2089.

8. Daar, E.S., et al. 2001. Diagnosis of primary HIV-1 infection. Ann. Intern. Med. 134:25-29.

9. Kinloch-de Loes, S., et al. 1993. Symptomatic primary infection due to human immunodeficiency virus type 1: review of 31 cases. Clin. Infect. Dis. 17:59-65.

10. Weintrob, A.C., et al. 2003. Infrequent diagnosis of primary human immunodeficiency virus infection: missed opportunities in acute care settings. Arch. Intern. Med. 163:2097-2100.

11. Rosenberg, E.S., Caliendo, A.M., and Walker, B.D.
1999. Acute HIV infection among patients tested for mononucleosis. N. Engl. J. Med. 340:969.

12. Pincus, J.M., et al. 2003. Acute human immunodeficiency virus infection in patients presenting to an urban urgent care center. Clin. Infect. Dis. 37:1699-1704.

13. Fiebig, E.W., et al. 2003. Dynamics of HIV viremia and antibody seroconversion in plasma donors: implications for diagnosis and staging of primary HIV infection. AIDS. 17:1871-1879.

14. Morandi, P.A., et al. 1998. Detection of human immunodeficiency virus type 1 (HIV-1) RNA in pools of sera negative for antibodies to HIV-1 and HIV-2. J. Clin. Microbiol. 36:1534-1538.

15. Quinn, T.C., et al. 2000. Feasibility of pooling sera for HIV-1 viral RNA to diagnose acute primary HIV-1 infection and estimate HIV incidence. AIDS. 14:2751-2757.

16. Pilcher, C.D., et al. 2002. Real-time, universal 
screening for acute HIV infection in a routine HIV counseling and testing population. JAMA. 288:216-221.

17. Weintrob, A.C., et al. 2003. Prevalence of undiagnosed HIV infection in febrile patients presenting to an emergency department in Southeastern United States. Program and abstracts, 2nd International AIDS Society Pathogenesis Meeting.Paris, France. Abstr. 84.

18. Clark, S.J., et al. 1994. Unsuspected primary human immunodeficiency virus type 1 infection in seronegative emergency department patients. J. Infect. Dis. 170:194-197.

19. Deng, H., et al. 1996. Identification of a major coreceptor for primary isolates of HIV-1. Nature. 381:661-666.

20. Pope, M., and Haase, A.T. 2003. Transmission, acute HIV-1 infection and the quest for strategies to prevent infection. Nat. Med. 9:847-852.

21. Geijtenbeek, T.B., et al. 2000. DC-SIGN, a dendritic cell-specific HIV-1-binding protein that enhances trans-infection of T cells. Cell. 100:587-597.

22. Zhang, Z., et al. 1999. Sexual transmission and propagation of SIV and HIV in resting and activated CD4+ T cells. Science. 286:1353-1357.

23. Schacker, T., et al. 2000. Rapid accumulation of human immunodeficiency virus (HIV) in lymphatic tissue reservoirs during acute and early HIV infection: implications for timing of antiretroviral therapy. J. Infect. Dis. 181:354-357.

24. Spira, A.I., et al. 1996. Cellular targets of infection and route of viral dissemination after an intravaginal inoculation of simian immunodeficiency virus into rhesus macaques. J. Exp. Med. 183:215-225.

25. Bogers, W.M., et al. 1998. Characteristics of primary infection of a European human immunodeficiency virus type 1 clade $B$ isolate in chimpanzees. J. Gen. Virol. 79:2895-2903.

26. Pilcher, C.D., et al. 2001. HIV in body fluids during primary HIV infection: implications for pathogenesis, treatment and public health. AIDS. 15:837-845.

27. Pilcher, C.D., et al. 2004. Brief but efficient: acute HIV infection and the sexual transmission of HIV. J. Infect. Dis. In press.

28. Koup, R.A., et al. 1994. Temporal association of cellular immune responses with the initial control of viremia in primary human immunodeficiency virus type 1 syndrome. J. Virol. 68:4650-4655.

29. Douek, D.C., et al. 2002. HIV preferentially infects HIV-specific CD4+ T cells. Nature. 417:95-98.

30. Shugars, D.C., Alexander, A.L., Fu, K., and Freel, S.A. 1999. Endogenous salivary inhibitors of human immunodeficiency virus. Arch. Oral Biol. 44:445-453

31. Shugars, D.C., and Wahl, S.M. 1998. The role of the oral environment in HIV-1 transmission. J. Am. Dent. Assoc. 129:851-858.

32. Roos, M.T., et al. 1992. Viral phenotype and immune response in primary human immunodeficiency virus type 1 infection. J. Infect. Dis. 165:427-432.

33. Zhu, T., et al. 1993. Genotypic and phenotypic characterization of HIV-1 patients with primary infection. Science. 261:1179-1181.

34. Wolinsky, S.M., et al. 1996. Adaptive evolution of human immunodeficiency virus-type 1 during the natural course of infection. Science. 272:537-542.

35. Ritola, K., et al. 2004. HIV-1 V1/V2 and V3 env diversity during primary infection suggests a role for multiply infected cells in transmission. Program and abstracts: 11th Conference on Retroviruses and Opportunistic Infections. San Francisco, California, USA. Abstr. 386.

36. Chun, T.W., et al. 1995. In vivo fate of HIV-1-infected T cells: quantitative analysis of the transition to stable latency. Nat. Med. 1:1284-1290.

37. Blankson, J.N., et al. 2000. Biphasic decay of latently infected CD4+ $\mathrm{T}$ cells in acute human immun- odeficiency virus type 1 infection. J. Infect. Dis. 182:1636-1642.

38. Zhang, L., et al. 1999. Quantifying residual HIV-1 replication in patients receiving combination antiretroviral therapy. N. Engl. J. Med. 340:1605-1613.

39. Lori, F., et al. 1999. Treatment of human immunodeficiency virus infection with hydroxyurea, didanosine, and a protease inhibitor before seroconversion is associated with normalized immune parameters and limited viral reservoir. J. Infect. Dis. 180:1827-1832.

40. Lafeuillade, A., Poggi, C., Hittinger, G., Counillon, E., and Emilie, D. 2003. Predictors of plasma human immunodeficiency virus type 1 RNA control after discontinuation of highly active antiretroviral therapy initiated at acute infection combined with structured treatment interruptions and immune-based therapies. J. Infect. Dis. 188:1426-1432.

41. Letvin, N.L., and Walker, B.D. 2003. Immunopathogenesis and immunotherapy in AIDS virus infections. Nat. Med. 9:861-866.

42. Henrard, D.R., et al. 1995. Virologic and immunologic characterization of symptomatic and asymptomatic primary HIV-1 infection. J. Acquir. Immune Defic. Syndr. Hum. Retrovirol. 9:305-310.

43. Rybarczyk, B.J., et al. 2004. Correlation between $e n v \mathrm{~V} 1 / \mathrm{V} 2$ region diversification and neutralizing antibodies during primary infection by simian immunodeficiency virus $\mathrm{sm}$ in rhesus macaques. J. Virol. In press.

44. Wei, X., et al. 2003. Antibody neutralization and escape by HIV-1. Nature. 422:307-312.

45. Richman, D.D., Wrin, T., Little, S.J., and Petropoulos, C.J. 2003. Rapid evolution of the neutralizing antibody response to HIV type 1 infection. Proc. Natl. Acad. Sci. U. S. A. 100:4144-4149.

46. Koup, R.A., et al. 1994. Temporal association of cellular immune responses with the initial control of viremia in primary human immunodeficiency virus type 1 syndrome. J. Virol. 68:4650-4655.

47. Schmitz, J.E., et al. 1999. Control of viremia in simian immunodeficiency virus infection by CD8+ lymphocytes. Science. 283:857-860.

48. Goulder, P.J., et al. 2001. Evolution and transmission of stable CTL escape mutations in HIV infection. Nature. 412:334-338.

49. Malhotra, U., et al. 2001. Role for HLA class II molecules in HIV-1 suppression and cellular immunity following antiretroviral treatment. J. Clin. Invest. 107:505-517.

50. Gao, X., et al. 2001. Effect of a single amino acid change in MHC class I molecules on the rate of progression to AIDS. N. Engl. J. Med. 344:1668-1675.

51. Kaslow, R.A., et al. 1996. Influence of combinations of human major histocompatibility complex genes on the course of HIV-1 infection. Nat. Med. 2:405-411.

52. Rosenberg, E.S., et al. 1997. Vigorous HIV-1-specific $\mathrm{CD} 4^{+} \mathrm{T}$ cell responses associated with control of viremia. Science. 278:1447-1450.

53. Pitcher, C.J., et al. 1999. HIV-1-specific CD4+ T cells are detectable in most individuals with active HIV-1 infection, but decline with prolonged viral suppression. Nat. Med. 5:518-525.

54. Rosenberg, E.S., et al. 2000. Immune control of HIV-1 after early treatment of acute infection. Nature. 407:523-526.

55. Lisziewicz, J., et al. 1999. Control of HIV despite the discontinuation of antiretroviral therapy. N. Engl.J. Med. 340:1683-1684.

56. Kilby, J., et al. 2000. Recurrence of the acute HIV syndrome after interruption of antiretroviral therapy in a patient with chronic HIV infection: a case report. Ann. Intern. Med. 133:435-438.

57. Kaufmann, D., et al. 2004. Limited durability of immune control following treated acute HIV infection. Program and abstracts: 11 th Conference on Retro- viruses and Opportunistic Infections. San Francisco, California, USA. Abstr. 24

58. Montefiori, D.C., et al. 2003. Viremia control despite escape from a rapid and potent autologous neutralizing antibody response after therapy cessation in an HIV-1-infected individual. J. Immunol. 170:3906-3914.

59. Perelson, A.S., et al. 1997. Decay characteristics of HIV-1-infected compartments during combination therapy. Nature. 387:188-191.

60. Lafeuillade, A., et al. 2001. Impact of immune interventions on proviral HIV-1 DNA decay in patients receiving highly active antiretroviral therapy. HIV Med. 2:189-194.

61. Kulkosky, J., et al. 2002. Intensification and stimulation therapy for human immunodeficiency virus type 1 reservoirs in infected persons receiving virally suppressive highly active antiretroviral therapy. J. Infect. Dis. 186:1403-1411.

62. Coull, J.J., et al. 2002. Targeted derepression of the human immunodeficiency virus type 1 long terminal repeat by pyrrole-imidazole polyamides. J. Virol. 76:12349-12354.

63. Oxenius, A., et al. 2000. Early highly active antiretroviral therapy for acute HIV-1 infection preserves immune function of CD8+ and CD4+ T lymphocytes. Proc. Natl. Acad. Sci. U. S. A. 97:3382-3387.

64. Malhotra, U., et al. 2000. Effect of combination antiretroviral therapy on T-cell immunity in acute human immunodeficiency virus type 1 infection. J. Infect. Dis. 181:121-131.

65. Otten, R.A., et al. 2000. Efficacy of postexposure prophylaxis after intravaginal exposure of pig-tailed macaques to a human-derived retrovirus (human immunodeficiency virus type 2). J. Virol. 74:9771-9775.

66. Galvin, S.R., and Cohen, M.S. 2004. The role of sexually transmitted diseases in HIV transmission. Nat. Rev. Microbiol. 2:33-42.

67. Hogan, C.M., and Hammer, S.M. 2001. Host determinants in HIV infection and disease. Part 2: genetic factors and implications for antiretroviral therapeutics. Ann. Intern. Med. 134:978-996.

68. Fleming, D., and Wasserheit, J. 1999. From epidemiological synergy to public health policy and practice: the contribution of other sexually transmitted diseases to sexual transmission of HIV infection. Sex. Transm. Infect. 75:3-17.

69. Rottingen, J.A., Cameron, D.W., and Garnett, G. 2001. A systematic review of the epidemiologic interactions between classic sexually transmitted diseases and HIV. Sex. Transm. Dis. 28:579-597.

70. Taha, T.E. 1998. Bacterial vaginosis and disturbances of vaginal flora: association with increased acquisition of HIV. AIDS. 12:1699-1706.

71. Gray, R.H. 2000. Male circumcision and HIV acquisition and transmission: cohort studies in Rakai, Uganda. AIDS. 14:2371-2381.

72. Quinn, T.C. 2000. Viral load and heterosexual transmission of human immunodeficiency virus type 1. N. Engl. J. Med. 342:921-929.

73. Hoffman, I.F. 1999. The effect of Plasmodium falciparum malaria on HIV-1 RNA blood plasma concentrations. AIDS. 13:487-494.

74. Vernazza, P.L., et al. 1994. Detection and biologic characterization of infectious HIV-1 in semen of seropositive men. AIDS. 8:1325-1329.

75. Dyer, J., et al. 1996. Quantitation of human immunodeficiency virus type 1 RNA in cell-free seminal plasma: comparison of NASBA with Amplicor reverse transcription-PCR amplification and correlation with quantitative culture. J. Virol. Methods. 60:161-170.

76. Vernazza, P.L., et al. 1997. Quantification of HIV in semen: correlation with antiviral treatment and immune status. AIDS. 11:987-993.

77. Hart, C. 1999. Correlation of human immunodeficiency virus type 1 RNA levels in blood and female 
genital tract. J. Infect. Dis. 179:871-882.

78. Eron, J., Gilliam, B., Fiscus, S., Dyer, J., and Cohen, M. 1996. HIV-1 shedding and chlamydial urethritis. JAMA. 275:36.

79. Cohen, M.S., et al. 1997. Reduction of concentration of HIV-1 in semen after treatment of urethritis: implications for prevention of sexual transmission of HIV-1. AIDSCAP Malawi Research Group. Lancet. 349:1868-1873.

80. Wawer, M.J., et al. 2003. HIV-1 transmission per coital act, by stage of HIV infection in the index partner, in discordant couples, Rakai, Uganda. Program and abstracts: 10th Conference on Retroviruses and Opportunistic Infections. Boston, Massachusetts, USA. Abstr. 40.

81. Dyer, J.R., et al. 1997. Shedding of HIV-1 in semen during primary infection. AIDS. 11:543-545.

82. Pullium, J.K., et al. 2001. Pig-tailed macaques infected with human immunodeficiency virus (HIV) type 2GB122 or simian/HIV89.6p express virus in semen during primary infection: new model for genital tract shedding and transmission. J. Infect. Dis. 183:1023-1030.

83. Chakraborty, H., et al. 2001. Viral burden in genital secretions determines male-to-female sexual transmission of HIV-1: a probabilistic empiric model. AIDS. 15:621-627.

84. Zhu, T., et al. 1993. Genetic characterization of human immunodeficiency virus type 1 in blood and genital secretions: evidence for viral compartmentalization and selection during sexual transmission. J. Virol. 70:3098-3107.

85. Cameron, D.W., et al. 1989. Female to male transmission of human immunodeficiency virus type 1 : risk factors for seroconversion in men. Lancet. 2:403-407.

86. Colfax, G.N., et al. 2002. Sexual risk behaviors and implications for secondary HIV transmission during and after HIV seroconversion. AIDS. 16:1529-1535.

87. Jacquez, J.A., Koopman, J.S., Simon, C.P., and Longini, I.M., Jr. 1994. Role of the primary infection in epidemics of HIV infection in gay cohorts. J. Acquir. Immune Defic. Syndr. 7:1169-1184.

88. Koopman, J.S., et al. 1997. The role of early HIV infection in the spread of HIV through populations. J. Acquir. Immune Defic. Syndr. Hum. Retrovirol. 14:249-258.

89. Leynaert, B., Downs, A.M., and de Vincenzi, I. 1998. Heterosexual transmission of human immunodeficiency virus: variability of infectivity throughout the course of infection. European Study Group on Heterosexual Transmission of HIV. Am. J. Epidemiol. 148:88-96.

90. Yerly, S., et al. 2001. Acute HIV infection: impact on the spread of HIV and transmission of drug resistance. AIDS. 15:2287-2292.

91. Clumeck, N., et al. 1989. A cluster of HIV infection among heterosexual people without apparent risk factors. N. Engl. J. Med. 321:1460-1462.

92. Ruiz, M.S., et al., editors. 2000. No time to lose: getting more from HIV prevention. National Academies Press. Washington, DC, USA. 227 pp.

93. Alvarez-Darder, C., Marrques, S., and Perea, E.J. 1985. Urban cluster of sexually transmitted diseases in the city of Seville, Spain. Sex. Transm. Dis. 12:166-168.

94. Rothenberg, R.B. 1983. The geography of gonorrhea: empirical demonstration of core group transmission. Am. J. Epidemiol. 117:688-694.

95. Janssen, R.S., et al. 1998. New testing strategy to detect early HIV-1 infection for use in incidence estimates and for clinical and prevention purposes [erratum 1999, 281:1893]. JAMA. 280:42-48.

96. Foust, E., et al. 2003. Partner counseling and referral services to identify persons with undiagnosed HIV: North Carolina, 2001. MMWR Morb. Mortal. Wkly. Rep. 52:1181-1184.

97. Rothenberg, R.B., et al. 1998. Using social network and ethnographic tools to evaluate syphilis transmission. Sex. Transm. Dis. 25:154-160.

98. Luster, A.D. 1998. Chemokines: chemotactic cytokines that mediate inflammation. N. Engl. J. Med. 338:436-445.

99. Kahn, L.O., and Walker, B.D. 1998. Acute human immunodeficiency virus type 1 infection. N. Engl.J. Med. 339:33-39. 